\title{
Costing Mixed Coxian Phase-type Systems in a given time interval
}

\author{
Sally McClean ${ }^{1}$, Lalit Garg ${ }^{2}$, Jennifer Gillespie ${ }^{1}$, Ken Fullerton ${ }^{3}$ \\ ${ }^{1}$ School of Computing and Information Engineering, University of Ulster, Coleraine, UK \\ ${ }^{2}$ Computer Information Systems, University of Malta, Malta \\ ${ }^{3}$ School of Medicine, Dentistry, and Biomedical Sciences, Queens University, Belfast, UK \\ si.mcclean@ulster.ac.uk; lalit.garg@um.edu.mt; ken.fullerton@belfasttrust.hscni.net
}

\begin{abstract}
Previously we have introduced a modelling framework to classify individuals in Mixed Coxian Phase-type Systems. We here add costs and obtain results for moments of total costs in $(0, t]$, for an individual, and a cohort arriving at time zero. Based on data from the Belfast City Hospital Stroke Unit we use the overall modelling framework to obtain results for total cost in a given time interval to facilitate planners who have limited time horizons for budget planning.
\end{abstract}

\section{Introduction}

This paper extends our previous continuous-time Markov modelling framework [9] that developed phasetype $(\mathrm{PH})$ models to describe lengths of stay (LOS) for individuals moving through a system (the transient states) prior to departure to an absorbing state. The aim is to provide moments of total costs for the system in a given time interval, for individuals and a cohort entering the system at time zero. When modelling and costing such systems it is important to consider that an individuals LOS may be heterogeneous with respect to various covariates $[9,7]$. Therefore, the current framework considers a system comprising of a mixture of PH models. The mixture components correspond to classes of individuals having different phase-type distributions (PHD) $[9,4,10,1,8]$. The individuals move through the $\mathrm{PH}$ transient states, incurring differential costs per unit time in each state; such transitions typically represent patient movements from acute, through diagnosis, treatment and rehabilitation to long-stay. A number of approaches have been used to cluster LOS data and generate patient classes [9]. We extend our previous work, which clusters patients LOS using survival trees into PH (Markov) models, to a cost model. Costing is specific to the particular pathway an individual follows. The approach is then used to model a stroke unit where patients are divided into classes, characterized by the covariates: gender, age, disease and diagnosis $[7,4,1]$. This can help provide better predictions of the patient LOS, and future requirements for hospital beds and other resources [4, 8]. Previously [9, 4], we have developed a mixture distribution for determining clinically meaningful patient groups from a given dataset of patients' LOS. Costs can also be assigned to each stage of each group and future costs estimated $[10,1]$. Moments of costs of patient care in future time periods can thus be estimated and used for patient prognostication and health service planning. The current paper also extends our previous result for the mean numbers of patients in future states [5]. In this paper we provide the Moment Generating Function (MGFs) of total cost in time $(0, \mathrm{t}]$ for an individual and a cohort of patients moving through a Mixed Coxian PHD. These functions are beneficial to policy makers who have limited time horizons for budgets, budget planning and provisioning for new interventions, such as thrombolysis. In Section 2 we provide background on Coxian PHDs and Mixed Coxian PHDs. In Section 3, we define a mixed Coxian $\mathrm{PH}$ system and derive novel expressions for the MGF of cost in $(0, t]$ of an individual and a cohort moving through a mixed Coxian $\mathrm{PH}$ system. In Section 4, the approach is used to cost stroke patient care in the Stroke Unit of the Belfast City Hospital $(\mathrm{BCH})$. Concluding remarks and a discussion of further work are provided in Section 5.

\section{Background}

\subsection{Coxian PHD}

PHDs are a class of distributions in which a random variable generated by one or more Markov stochastic process(es) is modelled as an absorbing Markov chain having $\mathrm{k}$ transient states and an absorbing state (Figure 1). Durations of PHDs are defined on the non-negative real numbers. A PHD comprises a number of phases with sub-durations which are exponentially distributed. An individual enters a state of the system, moves between transients states until 
eventually absorption occurs. A special type of PHDs is the Coxian PHD [2,3], which provides a simple interpretation of fit for the duration data and has many other advantages over other types of PHDs [3]. A Coxian PHD process starts in the first transient state and develops by either sequentially passing through the transient states or moving to the absorbing state; we here envisage such transitions as representing phases of treatment and care. Each transient state can be modelled by two parameters: rate of sequential transition to the next state $\left(\lambda_{i}\right)$ and rate of transition to the absorbing state $\left(\mu_{i}\right)$. The probability density function of a Coxian PHD with duration $x$ is: $f_{P H D}(x)=\mathbf{p} \exp \mathbf{Q} x \mathbf{q}$, where $\mathbf{Q}$ is the transition matrix and, for $k$ transient states it is defined as follows:

$$
\mathbf{Q}=\left(\begin{array}{ccccc}
-\left(\lambda_{1}+\mu_{1}\right) & \lambda_{1} & 0 & \ldots & 0 \\
0 & -\left(\lambda_{2}+\mu_{2}\right) & \ddots & \ddots & \vdots \\
0 & 0 & \ddots & \ddots & 0 \\
\vdots & \ddots & \ddots & \ddots & \lambda_{k-1} \\
0 & \ldots & 0 & 0 & -\mu_{k}
\end{array}\right)
$$

The row admission vector $\mathbf{p}$, represents the initial state probability distribution and is defined as $\mathbf{p}=(1,0, \ldots, 0)$. The column discharge vector $\mathbf{q}$ represents the absorption probabilities and is defined as $\mathbf{q}=\left(\mu_{1}, \mu_{2}, \ldots, \mu_{k}\right)^{\prime}$ Also, $-\mathbf{Q}^{-\mathbf{1}} \mathbf{q}=\mathbf{e}$, where $\mathbf{e}=(1,, 1)^{\prime}$ is a $k \times 1$ column vector. For a non-defective PHD, starting from any transient

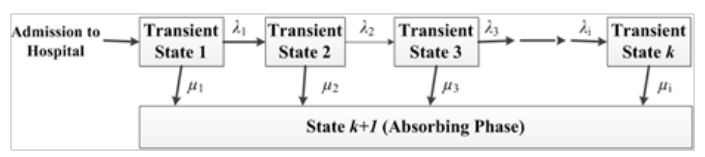

Figure 1: System described as a $k$ state Coxian PHD.

state, absorption occurs with probability 1 [6]. The matrix $\mathbf{P}(t)=P_{i j}(t)=\exp (\mathbf{Q} t)$ is the matrix of transition probabilities from transient state $S_{i}$ to transient state $S_{j}$ in $(0, t]$; therefore, $\lim _{t \rightarrow \infty} \exp (\mathbf{Q} t)=0$. For non-defective PHDs, the matrix $\mathbf{Q}$ is non-singular [6] and all possible paths starting from a transient state lead to the absorbing state. This is always true for a Coxian PH model with $\lambda_{i}+\mu_{i}>0$ for $i=1 \ldots, k$ (defining $\lambda_{k}=0$ ). Without loss of generality we therefore assume that our PHD is non-defective and hence $\mathbf{Q}$ is non-singular.

\subsection{Mixed Coxian PHD}

Here we use mixtures of Coxian PHDs, since they allow us to describe systems where individuals choose one of the mixture components with a given probability; they then follow a Coxian PHDs as illustrated in Figure 1, where each mixture component may correspond to a Coxian with different parameters. We therefore define $C$ mixture components, where there are $k_{c}$ phases (states) in class $c$, for $c=1, \ldots, C$ phases (states) in all, and $k=\sum_{c=1}^{C} k_{c}$.

Transitions occur from state $S_{i}^{c}\left(i=1, \ldots, k_{c-1}\right)$ to state $S_{i+1}^{c}$ with transition rate $\lambda_{i j}^{c}$ and transition is never possible between a transient state in any class and another transient state in a different class. Also transition can occur from any state $S_{i}^{c}$ of class $c$ to the absorbing state $S_{k+1}$ with transition rate $\mu_{i, k+1}^{c}$, for $i=1, \ldots, k_{c}, c=1, \ldots, C$. The admission vector $\mathbf{p}$ is now a row vector, partitioned into $\mathrm{C}$ subvectors (the mixture components), $\mathbf{p}=\left(\mathbf{p}_{\mathbf{1}}, \ldots, \mathbf{p}_{\mathbf{C}}\right)$. The $c$ th sub-vector $\mathbf{p}_{c}$ has first element $\pi_{c}$, which is the probability of entering phase 1 of the corresponding class, and the remaining elements of $p_{c}$ are zeros, for $c=1, \ldots, C$. Also, $\mathbf{q}=\left(\mathbf{q}_{\mathbf{1}}, \ldots, \mathbf{q}_{\mathbf{C}}\right)$ where the sub-vectors correspond to the classes. Each of the Coxian PHDs are non-defective since all possible paths, starting from a transient state in class $\mathrm{c}$, leads to an absorbing state. The transition matrix $\mathbf{Q}$ is now given by:

$$
\begin{aligned}
& \mathbf{Q}=\left(\begin{array}{cccc}
\mathbf{Q}_{1} & 0 & \ldots & 0 \\
0 & \mathbf{Q}_{2} & \ddots & \vdots \\
\vdots & \ddots & \ddots & 0 \\
0 & \ldots & 0 & \mathbf{Q}_{C}
\end{array}\right) \text { where } \\
& \mathbf{Q}_{C}=\left(\begin{array}{ccccc}
-\left(\lambda_{1}^{C}+\Sigma \mu_{1 j}^{C}\right) & \lambda_{1}^{C} & 0 & \ldots & 0 \\
0 & -\left(\lambda_{2}^{C}+\Sigma \mu_{2 j}^{C}\right) & \ddots & \ddots & \vdots \\
0 & 0 & \ddots & \ddots & 0 \\
\vdots & \ddots & \ddots & \ddots & \lambda_{k_{c}-1}^{C} \\
0 & \ldots & 0 & 0 & -\Sigma \mu_{k_{c} j}^{C}
\end{array}\right)
\end{aligned}
$$

This mixed Coxian PH structure retains the advantages of Coxian PHDs, including: the progression through successive states, and the computational aspects. Therefore, we can identify the mixture components using relevant covariates and fit the model to each component separately.

\section{Costing mixed Coxian PH systems}

We are interested in costing the mixed Coxian PH system discussed in the previous section. We extend our mixed Coxian PH models to a situation where there is a unit cost for phase $i$ of class $c$ for $i=1, \ldots, k_{c}, c=1, \ldots, C$. Also we define:

$$
\mathbf{B}=\left(\begin{array}{cccc}
\mathbf{B}_{1} & 0 & \ldots & 0 \\
0 & \mathbf{B}_{2} & \ddots & \vdots \\
\vdots & \ddots & \ddots & 0 \\
0 & \ldots & 0 & \mathbf{B}_{C}
\end{array}\right)
$$

where $\mathbf{B}_{C}$ is the cost matrix for class $C$. We define an expression for the MGF of total cost incurred in the transient 
states in $(0, t]$ for an individual and then for a cohort of $\mathbf{N}$ such individuals.

Theorem 1: The MGF of total cost $C(t)$ in $(0, t]$ for an individual entering a mixed $\mathrm{PH}$ system is given by:

$$
\begin{array}{r}
M_{C}(\theta ; t)=\mathbf{p}\{\exp (\mathbf{Q}+\theta \mathbf{B}) t-\mathbf{I}\}(\mathbf{Q}+\theta \mathbf{B})^{-1} \mathbf{q} \\
+\mathbf{p}\{\exp (\mathbf{Q}+\theta \mathbf{B}) t\} \mathbf{e}
\end{array}
$$

Therefore; $M_{C}^{\prime}(0 ; t)=E[C]=-\mathbf{p}\{\mathbf{I}-\exp (\mathbf{Q} t)\} \mathbf{Q}^{-1} \mathbf{B e}$

$$
\text { and } \begin{aligned}
M_{C}^{\prime \prime}(0 ; t)=E\left[C^{2}\right]= & 2 \mathbf{p}\{\mathbf{I}-\exp (\mathbf{Q} t)\}\left(\mathbf{Q}^{-\mathbf{1}} \mathbf{B}\right)^{2} \mathbf{e} \\
& +2 \mathbf{p}\{\exp (\mathbf{Q}) \mathbf{t}\}(\mathbf{B t}) \mathbf{Q}^{-\mathbf{1}} \mathbf{B e}
\end{aligned}
$$

Proof:

$$
\begin{array}{r}
M_{C}(\theta ; t)=E\left[e^{\theta c t} \mid t\right]=\int_{0}^{t} \mathbf{p} \exp ((\mathbf{Q}+\theta \mathbf{B}) s) \mathbf{q} d s \\
+\int_{t}^{\infty} \mathbf{p} \exp (\mathbf{Q} s+\theta \mathbf{B} t) \mathbf{q} d s \\
=\mathbf{p}\{\exp (\mathbf{Q}+\theta \mathbf{B}) t-\mathbf{I}\}(\mathbf{Q}+\theta \mathbf{B})^{-1} \mathbf{q} \\
+\mathbf{p}\{\exp (\mathbf{Q}+\theta \mathbf{B}) t\} \mathbf{e}
\end{array}
$$

The mean cost is then obtained by differentiating and setting $\theta=0$. Therefore,

$$
\begin{array}{r}
E[C(t)]=M_{C}^{\prime}(0 ; t)=-\mathbf{p}\{\mathbf{I}-\exp (\mathbf{Q t})\}(\mathbf{B} t) \mathbf{e} \\
+\mathbf{p}\{\exp (\mathbf{Q} t)-\mathbf{I}\} \mathbf{Q}^{-1} \mathbf{B e}+\mathbf{p}\{\exp (\mathbf{Q} t)\}(\mathbf{B} t) \mathbf{e} \\
=-\mathbf{p}\{\mathbf{I}-\exp (\mathbf{Q} t)\} \mathbf{Q}^{-1} \mathbf{B e}
\end{array}
$$

By differentiating again and putting $\theta=0$, we obtain:

$$
\begin{aligned}
E\left[C(t)^{2}\right]= & 2 \mathbf{p}\{\mathbf{I}-\exp (\mathbf{Q} t)\}\left(\mathbf{Q}^{-1} \mathbf{B}\right)^{2} \mathbf{e} \\
& +2 \mathbf{p}\{\exp (\mathbf{Q} t)\}(\mathbf{B} t) \mathbf{Q}^{-1} \mathbf{B} \mathbf{e}
\end{aligned}
$$

The MGF for the total cost in $(0, t]$ for a cohort of $N$ individuals who all enter the system is given by:

$M_{C}(\theta, N ; t)=\left(M_{C}(\theta ; t)\right)^{N}=\left(-\mathbf{p}\{\mathbf{I}-\exp (\mathbf{Q} t)\} \mathbf{Q}^{-1} \mathbf{B q}\right)^{N}$

Differentiating and putting $\theta=0$ gives the moments, as:

$$
\begin{array}{r}
E\left[C_{N}(t)\right]=N\left(-\mathbf{p}\{\mathbf{I}-\exp (\mathbf{Q} t)\} \mathbf{Q}^{-1} \mathbf{B q}\right) \text { and } \\
E\left[C_{N}^{2}(t)\right]=N(N-2)\left(-\mathbf{p}\{\mathbf{I}-\exp (\mathbf{Q} t)\} b f Q^{-1} \mathbf{B e}\right)+ \\
N\left(2 \mathbf{p}\{\mathbf{I}-\exp (\mathbf{Q} t)\}\left(\mathbf{Q}^{-1} \mathbf{B}\right)^{2} \mathbf{e}+2 \mathbf{p}\{\exp (\mathbf{Q} t)\}(\mathbf{B} t) \mathbf{Q}^{-1} \mathbf{B e}\right.
\end{array}
$$

Example 1: We consider a system with two classes each with transient states $S_{1}$ and $S_{2}$ (Figure 2). These could represent drug therapy $\left(S_{1}\right)$ and no drug therapy $\left(S_{2}\right)$, where patients are initially assigned to $S_{1}$ and $S_{2}$ with probabilities $\pi_{1}$ and $\pi_{2}$ and are discharged from $S_{1}$ and $S_{2}$ at rates $\mu_{1}$ and $\mu_{2}$, respectively.

In this example, $\mathbf{p}$ is the admission vector so $\mathbf{p}=\left(\pi_{1}, \pi_{2}\right)$,

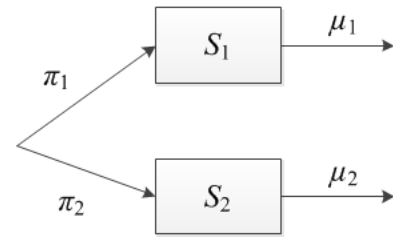

Figure 2: System with two classes, each containing one transient phase.

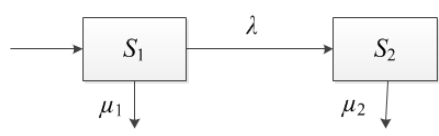

Figure 3: System with one class, containing two transient phases.

$\mathbf{Q}$ is the transition matrix given by:

$$
\mathbf{Q}=\left(\begin{array}{cc}
-\mu_{1} & 0 \\
0 & -\mu_{2}
\end{array}\right)
$$

and the rate at which patients leave each state is $\mathbf{q}=$ $\left(\mu_{1}, \mu_{2}\right)^{\prime}$. We also have costs for each transient state, where there is a cost $b_{1}$ per unit time in transient state $S_{1}$ and $b_{2}$ in transient state $S_{2}$ and $\mathbf{B}$ is given by:

$$
\mathbf{B}=\left(\begin{array}{cc}
b_{1} & 0 \\
0 & b_{2}
\end{array}\right)
$$

We can then calculate the mean cost in $(0, t]$. So,

$$
\begin{array}{r}
E[C(t)]=-\mathbf{p}\{\mathbf{I}-\exp (\mathbf{Q} t)\} \mathbf{Q}^{-1} \mathbf{B e} \\
=\left(b_{1} \pi_{1}\left(1-e^{-\mu_{1} t}\right)\right)\left(\mu_{1}\right)+\left(b_{2} \pi_{2}\left(1-e^{-\mu_{2} t}\right)\right)\left(\mu_{2}\right) \\
\text { and } E\left[C(t)^{2}\right]=2 \mathbf{p}\{\mathbf{I}-\exp (\mathbf{Q} t)\}\left(\mathbf{Q}^{-\mathbf{1}} \mathbf{B}\right)^{2} \mathbf{e} \\
+2 \mathbf{p}\{\exp (\mathbf{Q}) \mathbf{t}\}(\mathbf{B t}) \mathbf{Q}^{-\mathbf{1}} \mathbf{B e} \\
=\pi_{1}\left(1-e^{-\mu_{1} t}\right)\left(b_{1}^{2}\right)\left(\mu_{1}^{2}\right)+\pi_{2}\left(1-e^{-\mu_{2} t}\right)\left(b_{2}^{2}\right) /\left(\mu_{2}^{2}\right) \\
-2 \pi_{1} e^{-\mu_{1} t}\left(b_{1}^{2} t\right) /\left(\mu_{1}\right)-2 \pi_{2} e^{-\mu_{2} t}\left(b_{2}^{2} t\right) /\left(\mu_{2}\right)
\end{array}
$$

Example 2: We illustrate the theory using a system with one class which consists of two transient states $S_{1}$ and $S_{2}$ (Figure 3). These states could represent acute hospital care $\left(S_{1}\right)$ and long-stay hospital care $\left(S_{2}\right)$, where patients move from $S_{1}$ to $S_{2}$ at a rate $\lambda$ and leave $S_{1}$ and $S_{2}$ at rates $\mu_{1}$ and $\mu_{2}$, respectively.

In this example, $\mathbf{p}$ is the admission vector so $\mathbf{p}=(1,0)$, $\mathbf{Q}$ is the transition matrix given by:

$$
\mathbf{Q}=\left(\begin{array}{cc}
-\left(\lambda+\mu_{1}\right) & 0 \\
0 & -\mu_{2}
\end{array}\right)
$$

and the rate at which patients leave each state is $\mathbf{q}=$ $\left(\mu_{1}, \mu_{2}\right)^{\prime}$. We also have costs for each transient state, where 
there is a cost $b_{1}$ per unit time in transient state $S_{1}$ and $b_{2}$ in transient state $S_{2}$ and $\mathbf{B}$ is the same as in Example 2. We then calculate the mean cost in $(0, t]$ given by:

$$
\begin{array}{r}
E[C(t)]=-\mathbf{p}\{\mathbf{I}-\exp (\mathbf{Q} t)\} \mathbf{Q}^{-1} \mathbf{B} \mathbf{e} \\
=b_{1} /\left(\lambda+\mu_{1}\right)+\lambda b_{2} /\left(\lambda+\mu_{1}\right) \mu_{2}
\end{array}
$$

which we interpret as the unit cost in phase $1\left(b_{1}\right)$ multiplied by the mean duration in phase $1\left(1 /\left(\lambda+\mu_{1}\right)\right)$ plus the probability of progressing to phase $2\left(\lambda /\left(\lambda+\mu_{1}\right)\right)$ multiplied by unit cost in phase $2\left(b_{2}\right)$ and the mean duration in phase $2\left(1 / \mu_{2}\right)$.

\section{A Healthcare Application}

Our healthcare application is extracted from the Patient Administration System (PAS) and consists of all patients admitted to the BCH between 1 January, 2003 and 31 December 2007 with a diagnosis of stroke. Stroke patient care in $\mathrm{BCH}$ is provided by a Stroke Unit where the patient undergoes a period of acute care followed by a period of rehabilitation, if required, prior to discharge. The LOS distribution of each class was modelled using a PH model, starting with one state (exponential) and progressively increasing the number of states until, using a penalized likelihood approach, an optimal number of phases was determined. For our proposed PH model one or two phases were sufficient. The costs were 164.80 per day for acute care (state 1) and 114.8 per day for long-term care (state 2). The matrix B is therefore a diagonal $34 \times 34$ matrix with alternative elements 164.80 and 114.8 respectively. Results for mean co-

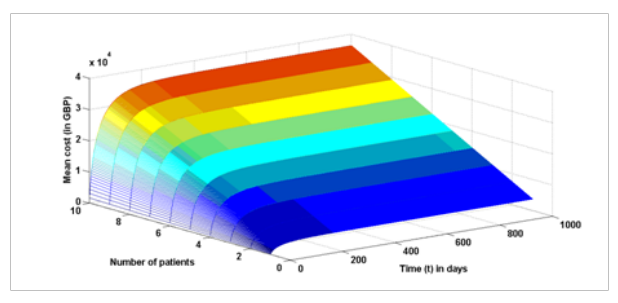

Figure 4: Mean cohort costs by time.

hort costs in $(0, t]$ are presented in Figure 4 for $t=0$ to 1000 days and cohort sizes 1 to 10 patients. We see that the cost is mainly incurred at the start of the period when most of the discharges occur. As t increases, increasingly less new costs are incurred as most patients have been discharged.

\section{Conclusion and Future Work}

Our current approach uses mixed Coxian PH systems to derive new expressions for MGFs and moments of cost, both for the individual and for cohorts who are admitted at time zero. Here, we focus on the Coxian mixture model as it allows us to tackle the problem of heterogeneity of durations in different states. Such a mixture analysis is an effective approach to prediction of costs in Markov systems where groups of individuals follow heterogeneous pathways. It is thus a powerful method for determining the relationship between input covariates and outcome. Currently we are extending our model to capacity and resource planning in a stroke care unit with Poisson admissions.

\subsection{Acknowledgements}

The authors acknowledge support for this research from the EPSRC for the MATCH project (Grant Reference GR/S29874/01).

\section{References}

[1] M. Barton, S. I. McClean, J. Gillespie, L. Garg, D. Wilson, and K. Fullerton. Is it Beneficial to Increase the Provision of Thrombolysis?-A Discrete-Event Simulation. QJM: An International Journal of Medicine, 105(7):665-673, 2012.

[2] D. Cox. A Use of complex probabilities in the theory of stochastic processes. Proceedings of the Cambridge Philosophical Society, 51(3):313-319, 1955.

[3] M. Fackrell. Modelling Healthcare Systems with Phase-type Distributions. Health Care Management Science, 12(1):1126, 2009.

[4] L. Garg, S. McClean, B. J. Meenan, E. El-Darzi, and P. H. Millard. Phase-Type survival trees and mixed distribution survival trees for clustering patients' length of stay. Informatica, 22(1):57-72, 2011.

[5] L. Garg, S. I. McClean, M. Barton, B. J. Meenan, and K. Fullerton. Intelligent Patient Management and Resource Planning for Complex, Heterogeneous, and Stochastic Healthcare Systems. IEEE Transactions on Systems, Man and Cybernetics-Part A:Systems and Humans, 42(6):13321345, 2012.

[6] G. Latouche and Z. Ramaswami. Introduction to Matrix Analytic Methods in Stochastic Modeling. SIAM Series onStatistics and Applied Probability, Philadelphia, 1999.

[7] A. H. Marshall and S. I. McClean. Using Coxian Phasetype Distributions to Identify Patient Characteristics for Duration of Stay in Hospital. Health Care Management Science, 7(4):285-289, 2004.

[8] A. H. Marshall, S. I. McClean, C. M. Shapcott, and P. H. Millard. Modelling Pateint Duration of Stay to Facilitate Resource Management of Geriatric Hospitals. Health Care Management Science, 5(4):313-319, 2002.

[9] S. I. McClean, M. Barton, L. Garg, and K. Fullerton. Combining Markov Models and Discrete Event Simulation to plan Stroke Patient Care. Transactions on Modeling and Computer Science, 21(4):Article 25, 2011.

[10] S. I. McClean, J. Gillespie, B. Scotney, and K. Fullerton. using Phase-type Models to cost a cohort of Stroke Patients. In 25th International Symposium on Computer-Based Medical Systems, 2012. 